\title{
Dynamics in Self-assembled Organic Monolayers at the Liquid/Solid Interface Revealed by Scanning Tunneling Microscopy
}

\author{
Steven De Feyter*, Hong Xu, and Kunal Mali
}

\begin{abstract}
The liquid/solid interface provides an interesting medium for molecular self-assembly and scanning tunneling microscopy is the preferred technique to analyse the structural features of the surface-supported self-assembled monolayers in this medium. An interesting aspect is the phenomenon of molecular dynamics at the liquid/solid interface. In this mini-review, we report on our efforts and strategies to investigate and even induce molecular dynamics at the liquid/solid interface, bringing insight to various kinds of processes such as conformational, translational and adsorption/desorption dynamics.
\end{abstract}

Keywords: Dynamics · HOPG · Scanning tunneling microscopy · Self-assembly

\section{Introduction}

Scanning tunneling microscopy (STM) is a very useful technique to investigate molecular adsorption on atomically-flat conductive surfaces, with submolecular resolution. While initial efforts addressed the visualisation of molecules under ultrahigh vacuum (UHV) conditions, soon it became clear that such conditions are not an absolute requirement for high-quality microscopy and visualisation. In particular, the liquid/solid interface turned out to be an excellent medium to induce molecular self-assembly, typically resulting in the formation of surface-supported monolayers and to investigate their structural features by STM. ${ }^{[1,2]}$ Successful monolayer formation and STM imaging require balanced molecule-molecule and molecule-substrate interactions: a too strong interaction immobilises the molecules and impedes self-assembly into ordered two-dimensional (2D) layers. A too weak interaction may lead to a too high mobility making high-resolution STM imaging impossible.
Compared to sample preparation and measuring under UHV conditions, the liquid/solid interface has a number of advantages: i) The experimental approach is straightforward and does not require a complicated or as expensive infrastructure. ii) Though the UHV environment provides excellent control leading to unprecedented high resolution, ${ }^{[3-6]}$ not all species can be adapted to UHV, such as those with relatively low thermal stability or big size. The demands set on the properties (size and function) of the molecules under investigation at the liquid/solid interface are more relaxed. iii) The choice of solvent is flexible and can be tuned to suit a particular solute and/or substrate. Typically, the solvent has a low vapour pressure, is nonconductive (electrochemically inert) and shows a lower affinity for the substrate than the solute. As a result, the liquid/solid interface approach in combination with STM imaging is becoming increasingly popular to induce and investigate self-assembly on surfaces. ${ }^{[7-9]}$

Additional control of the monolayer formation can be achieved under potential control in aqueous solutions. Under electrochemical conditions, adsorbate-substrate interactions can be modulated by the surface charge density. Electrochemical environments offer therefore additional possibilities to control surface dynamics and monolayer structure via the surface charge and to image those structures by means of electrochemical scanning tunneling microscopy: EC-STM. ${ }^{[10-12]}$

For self-assembly at the liquid/solid interface, the liquid phase acts as a reservoir of dissolved species which can diffuse towards the substrate, adsorb, diffuse laterally and desorb. This spontaneous dynamics is arguably one of the main advantages of self-assembly at the liquid/solid interface. Of great importance is the fact that these dynamic processes favour the repair of defects. Moreover, large domains grow at the expense of small domains via a process called Ostwald ripening. The choice of solvent affects the mobility of molecules, especially, the adsorption-desorption dynamics via the solvation energy and possibly also via solvent viscosity. Going beyond self-assembly, a number of recent reports stress the importance of 'reversibility' to obtain high-quality 2D surfacesupported covalent organic frameworks, combining molecular self-assembly and organic chemistry, on substrates such as graphite or iodide-covered $\mathrm{Au}(111)$. [13,14]

Several exciting dynamic phenomena were discovered and discussed such as translational, ${ }^{[15-22]}$ rotational, ${ }^{[23-25]}$ and conformational dynamics ${ }^{[26-31]}$ or a combination thereof, of single molecules or clusters on atomically flat metallic surfaces. Sometimes, these dynamics were intentionally provoked by the interaction with the STM-tip. ${ }^{[32-42]}$

Compared to molecular systems adsorbed under UHV conditions, an extra hurdle in probing dynamics in physisorbed self-assembled monolayers at a liquid/solid interface is the fact that molecules are part of a monolayer and often packed in a 2D-lattice. The observation of molecular dynamics at the single molecule level using STM at liquid/solid interfaces imposes a number of challenges. This is because, under physisorption conditions, at room 
temperature, most low-molecular weight molecules are too mobile to be visualised, except if they are trapped in a 2D (crystalline) matrix. Thus, any vertical motion, e.g. the desorption of a molecule from the monolayer into the liquid phase and the adsorption of a molecule from the liquid phase into the monolayer at the same site, occurs very fast and goes normally unnoticed. Likewise, typically no spontaneous conformational changes at the single molecule level are observed in the 'bulk' of such molecular monolayers. Studies on spontaneous dynamics at the liquid/ solid interface mainly deal with Ostwald ripening, ${ }^{[43-45]}$ i.e. the growth of larger domains at the expense of smaller ones or the conversion of one polymorph (phase) into another polymorph (phase). ${ }^{[46,47]}$ These spontaneous processes involve (collective) changes in molecular orientation (translation/rotation) and possibly also conformation though the latter events have rarely been probed at the single molecule level. In this contribution, we review our efforts to observe or induce dynamics at the liquid/solid interface using STM not only as visualisation technique but also as an active probe.

For the non-specialist readers, it is important to realise that STM images are not just 'photographs'. Dynamics in a photograph often show up as blurred features. The larger the shutter time, the greater the chance of getting blurred images if the object or camera are not fixed. Not so for STM imaging. Considerable dynamics can take place, fast or slow, while still leading to very sharp and high-quality images. This can be understood as follows: in STM imaging, the metallic tip is scanned across the surface and depending on the size of the area and the scanning speed, it might take less than a second or several minutes to scan a surface, several seconds to tens of seconds being typical values. The molecular dynamics can be that fast, for instance hopping of molecules between two sites or conformational dynamics, that the obtained STM image is a superposition of several discrete situations: in the STM image, both sites seem to be occupied, or the molecules have a 'strange' appearance, the latter being the result of the non-resolved conformational dynamics. Basically, such effects arise if the scanning speed is much slower than the dynamics involved. Another potential effect of the slow scanning speed compared to the dynamic phenomena is the appearance of abrupt structural changes during the scan. For instance the structural features in that part of the image which is scanned first, might seem disconnected from the part scanned later, because of desorption of the monolayer or a structural reorganisation.

\section{Structural Reorganisation in Monolayers and the Role of Solvent}

A firm proof for adsorption dynamics and its impact on the monolayer structures is demonstrated by the following example. Upon dissolving 5-alkoxy-isophthalic acid derivatives in for instance 1-octanol and applying a droplet of this solution on top of the basal plane of highly oriented pyrolytic graphite (HOPG), a regular monolayer structure is observed. The 5-alkoxyisophthalic acid molecules organise in lamellae: along the long lamella axis, the orientation of the molecules alternates, leading to a pattern stabilised by alkyl chain interdigitation (lower right of Fig. 1A).[48] Adjacent lamellae are stabilised by hydrogen bonding interactions between the isophthalic acid groups. However, in some areas, the lamellae of 5-alkoxy-isophthalic acid are separated by what seems to be a row of solvent molecules, i.e. 1-octanol. It turned out to be possible to visualise the co-deposition process of 1-octanol molecules (Fig. 1A-C). [49] This image sequence shows insertion of 1-octanol molecules between two isophthalic acid lamellae. A domain boundary at the lower left corner of these STM images is used as a reference point. The arrows indicate the location in the monolayer where insertion of the 1-octanol molecules starts. As shown in the time-lapse STM images, the insertion process is completed within two minutes and involves the adsorption of $\sim 48$ molecules of 1-octanol. Not surprisingly, this process is initiated at a domain boundary, typically a region with lower stability and enhanced dynamics. Co-deposition involves the exchange of molecules with the supernatant solution and reorientation (translation) of large monolayer parts in a cooperative fashion.

\section{Adsorption-Desorption Dynamics}

In addition to the dynamics involving detailed structural reorganisation at do- main boundaries as described above, more subtle adsorption/desorption dynamics can take place, for instance in the bulk of a monolayer. In monocomponent systems, it is normally not possible to visualise the dynamics, except if the free site of a desorbed molecule is not immediately taken by one of the identical solute molecules in the supernatant solution. One way to observe these dynamics is by mixing structurally related molecules which differ from each other in their appearance in the STM images. For instance, in order to distinguish between alkylated isophthalic acid derivatives, we introduced semi-fluorinated isophthalic acid derivatives, which can act as markers.[50] Individual semifluorinated molecules are co-adsorbed in host lamellae formed by the alkylated isophthalic acid derivatives. The perfluorinated part of the alkyl chain can be clearly distinguished as a black band, due to the decreased tunneling current. For mixed solutions of semi-fluorinated and nonfluorinated isophthalic acid derivatives, it was observed that whether or not segregation takes place depends upon the difference in length between the semi-fluorinated and non-fluorinated molecules. ${ }^{[50]}$ If the chain length differs only by one methylene group, mixed monolayers are formed (e.g. H11F6 and H16F0, Fig. 2B). Predominantly the non-fluorinated molecules are adsorbed with some individual semi-fluorinated molecules co-deposited within the lamellae. Individual semi-fluorinated molecules are dispersed in a matrix formed by the non-fluorinated ones. Scanning at two frames per second, it was observed that semi-fluorinated molecules desorb and are replaced by non-fluorinated ones (H6F12 and H18F0, Fig. 2C-E). ${ }^{[49]}$ The residence time of a single fluorinated molecule is of the order of seconds to several minutes, much longer than the estimated residence time reported for mixtures of saturated/unsaturated acids and alcohol/thiol mixtures by Stevens et al..$^{[51]}$ When neglecting solvent effects, this can partially be accounted for by the stabilising
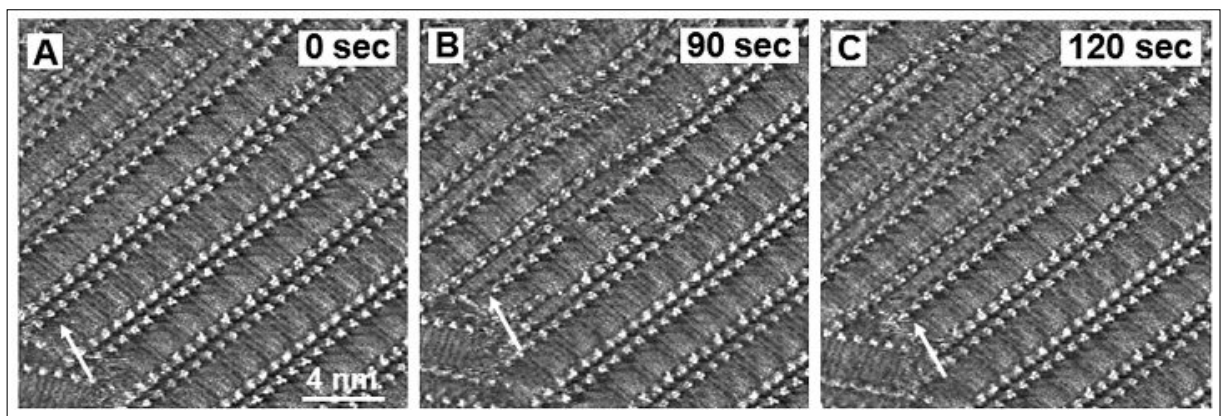

Fig. 1. Co-deposition dynamics of 1-octanol in a 5-icosyloxy-isophthalic acid monolayer. A sequence of STM images obtained by scanning the same area of the monolayer. White arrows indicate the location where the insertion of 1-octanol molecules between two adjacent lamellae is initiated. The wider lamellae are formed by isophthalic acid molecules whereas the narrower ones are built up by coadsorption of solvent molecules. 


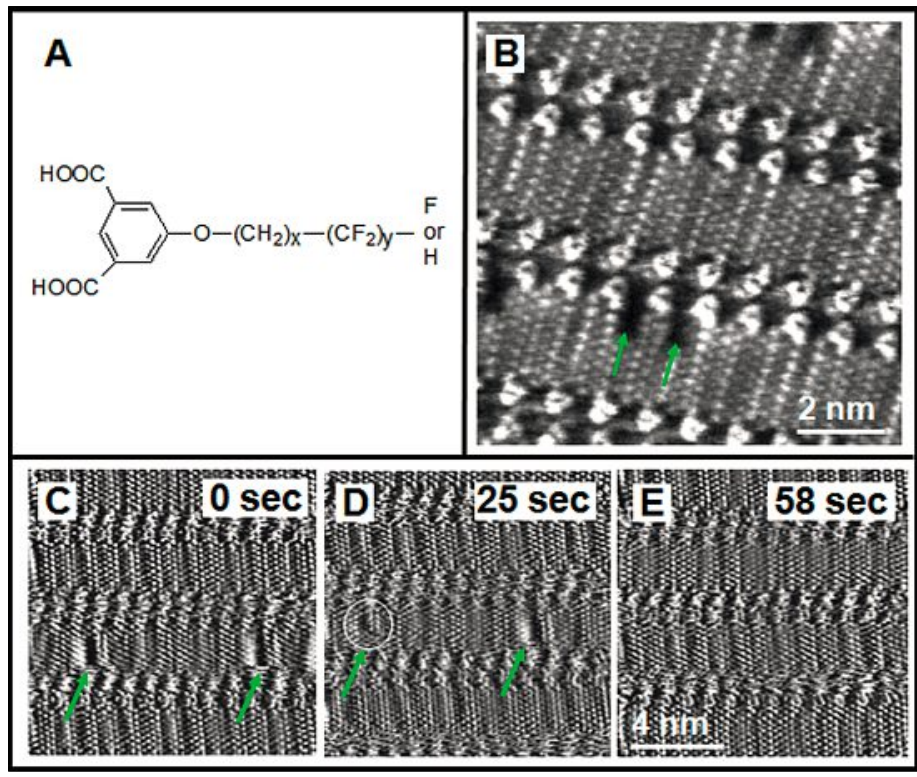

Fig. 2. A) Molecular model of 5-alkoxy-isophthalic acid and the semifluorinated analogue. B) Miscibility of H11F6 ( $x=11, y=6)$ and H16F0 $(x=16, y=0)$ molecules. The green arrows indicate H11F6 molecules. (C, D, E) Snapshots of the desorption of H6F12 $(x=6, y=12)$ molecules in $\mathrm{H} 18 \mathrm{FO}(\mathrm{x}=18, \mathrm{y}=0$ ) lamellae. The H6F12 molecules are indicated by arrows. The circle in $\mathrm{D}$ indicates a molecule with an apparent smaller fluorinated part. This is caused by desorption of a H6F11 molecule while the tip was scanning over the molecule.

effect of isophthalic acid hydrogen bonding. As anticipated, the stronger the molecule-molecule and molecule-substrate interactions, in combination with unfavourable solvation conditions (the choice of solvent!) the slower the dynamics. One should take this point into account in order to select a proper solvent.

\section{Conformational Dynamics}

Imaging conformational dynamics is a challenge that goes beyond the mere observation of adsorption-desorption dynamics. One of the reasons is that in low-molecular weight molecules adsorbed at the liquid/ solid interface and confined in a 2D lattice, the dynamics are probably rather fast, much faster than what can be recorded by STM. In order to slow down the dynamics, it appeared to us that it is necessary to come up with a large molecule. Moreover, in order to witness the dynamics, we realised that the 'moving' parts of the molecule

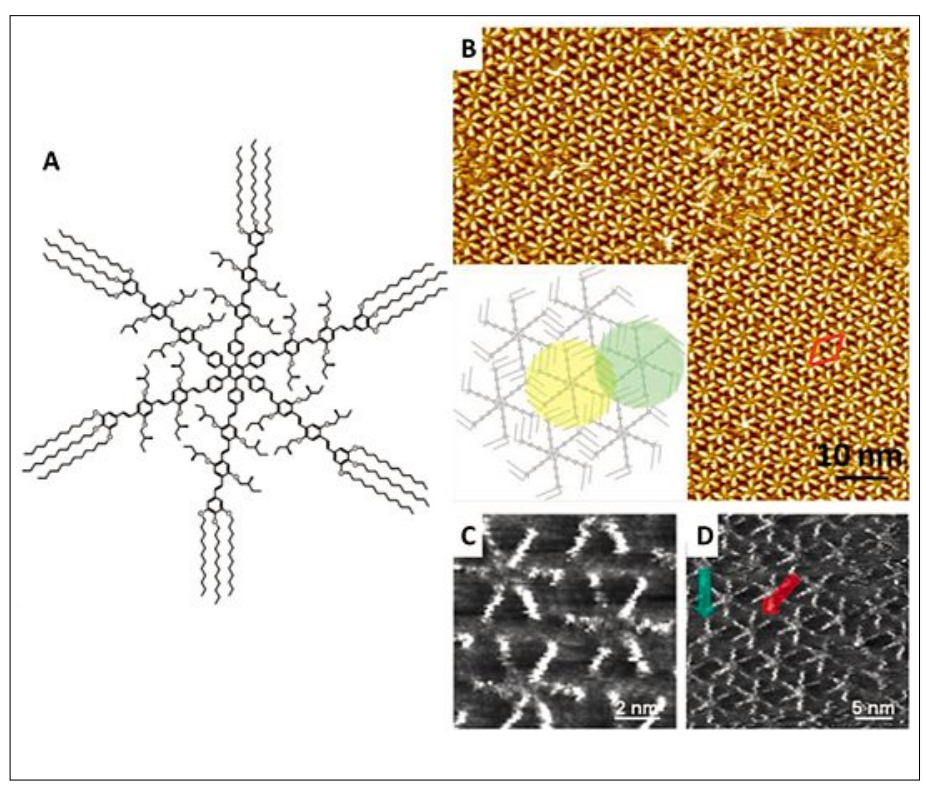

Fig. 3. A) Chemical structure of the six-legged oligo(p-phenylene vinylene)-substituted hexaarylbenzene 1. B) Large-scale STM image of monolayer of 1 physisorbed at the 1-phenyloctane/HOPG interface. Inset: Model reflecting the ordering of the molecules in a 2D crystal. Two alkyl chains per OPV leg are adsorbed. The third one, which is omitted in the model, is most likely solvated. The intersection of the two coloured disks coincides with the area of interaction between these two adjacent hexapods. A unit cell is indicated in red. C) Zoom showing the expected star-shape of the molecules. D) In addition to the 6-legged molecules, molecules are visible with apparently only five (red arrow) or even four legs (green arrow).

should be recognised in a straightforward fashion by the STM technique.

Therefore, we designed a six-legged oligo( $p$-phenylenevinylene) (OPV) substituted hexa-arylbenzene (1, Fig. 3A) that acts as molecular 'hexapod' in a self-assembled monolayer at the 1-phenyloctane/ HOPG interface. ${ }^{[52-54]}$

Structurally defect-free 2D crystalline domains covering large areas are not observed though often 'disordered' domains are observed in the midst of or between these small crystalline domains. We identify a group of molecules as belonging to a disordered domain if the centres of the molecules are not on top of 2D crystalline lattice points within a given area or if the molecules have a different orientation than those in the crystalline matrix. In total, the appearance of more than 20,000 molecules was evaluated by a human observer. About $2 / 3$ of these molecules self-assemble into a regularly ordered matrix whereas the rest are adsorbed in what we define as disordered domains.

Some of these molecules appear to have only five or four legs as illustrated in Fig. 3D. Strikingly, the situation in the so-called ordered and disordered domains is completely different. In ordered areas, most of the molecules appear as hexapods (96.0\%). 3.3\% of the molecules appear with five legs and only $0.7 \%$ with four legs. In contrast, this ratio differs drastically in disordered domains. Only $23 \%$ appear as hexapods, while the majority of the molecules $(57 \%)$ appear with five legs and $20 \%$ of the molecules in disordered domains show four legs.

Certain molecules show some interesting changes in appearance. Quite often, the number of 'legs' changes: legs disappear and reappear. Fig. 4 shows a series of images zooming in on the different appear-

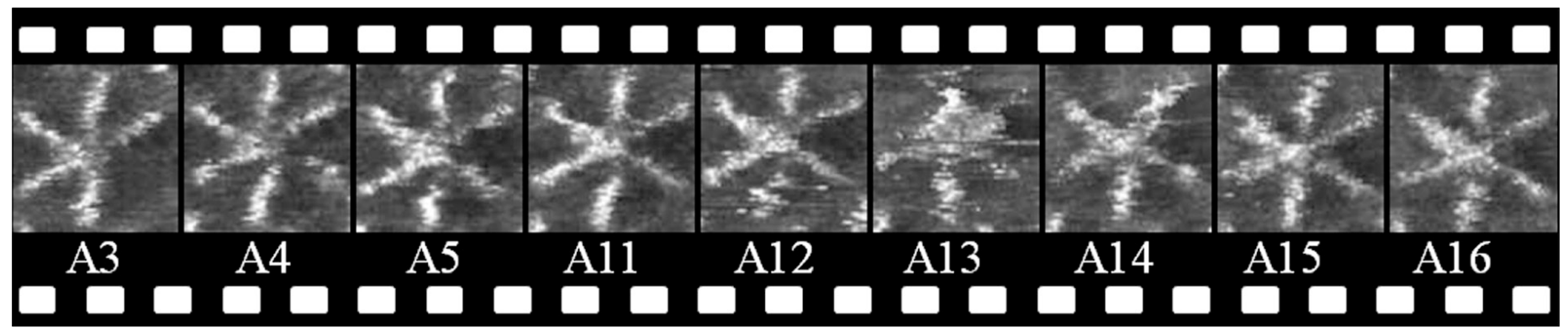

Fig. 4. Sequence of STM images zooming in on one molecule of 1 . The time difference between two consecutive frames $(e . g$. An $\rightarrow A(n+1))$ is about 14 seconds. 


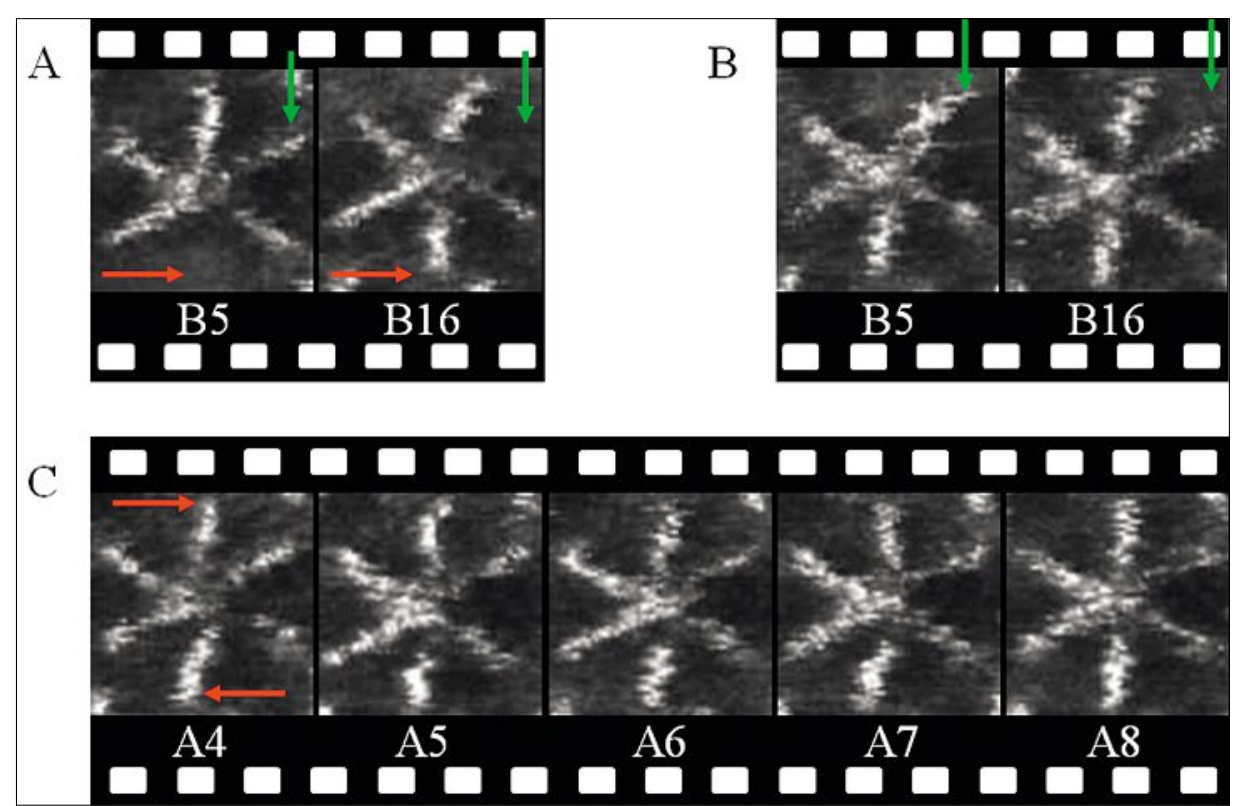

Fig. 5. Sequence of STM images zooming in on one molecule of $\mathbf{1}$ highlighting different dynamics. A) The molecule appears to rotate. At the position of the red arrow, a leg appears while at the position of the green arrow, a leg disappears. B) Re-adsorption of a leg, affecting the orientation of the leg originally at 1 o'clock. C) The legs indicated by the red arrows slightly change their orientation from frame to frame, though the overall orientation of the molecule remains unchanged. The frame numbers are indicated below each image.

ances of one molecule as a function of time. In frame A3, the molecule only shows five legs: the leg at 4 o'clock is missing, while in frame A4 all legs are visible. The image in frame A13 is blurred, and marks the transition from six visible legs (frame A12) to five visible legs (frame A14). In frame A15, all six legs appear again.

Not only does the number of visible legs change, also their orientation changes in time. Basically, two types of orientational changes can be observed. A first type involves a transition where the desorptionadsorption of legs results in an apparent rotation of the molecules. For instance, in Fig. $5 \mathrm{~A}$, at the position of the red arrow, a leg appears while at the position of the green arrow, a leg disappears. Overall, the molecule appears to be rotated. This rotation hypothesis is unlikely as it involves the desorption and adsorption of all legs. Note that the position of the other legs has not changed.

In addition, these molecules are not rigid as far as the position of the legs is concerned. The molecule in Fig. 5B undergoes a transition from five adsorbed legs to six adsorbed legs. Note, however, that the 're-adsorption' of the sixth leg follows the reorientation of the leg indicated in green: originally at 1 o'clock, it appears subsequently at the 12 o'clock position. Also more subtle orientational changes can be observed (Fig. 5C). The legs indicated by the red arrows slightly change their orientation from frame to frame, though the overall orientation of the molecule remains unchanged. The effect is that the angle between the legs is not always $60^{\circ}$ but can change considerably.
The disappearance and reappearance of the bright legs is the result of desorption/ re-adsorption or reorientation of legs. In addition, the larger than statistically predicted number of dimer defects in the $2 \mathrm{D}$ crystalline lattice indicates that molecules mutually interact and that desorption of a leg in a hexapod promotes the desorption of a leg in a hexapod next to it.

In the conformational dynamics discussed above, the position of the centre of mass of the molecules was not found to change. However, in addition to the conformational dynamics, translational motion was also observed, as highlighted in the three frames presented in Fig. 6. In this

\section{Translational Dynamics}

sequence of images, there is translational motion on the surface in areas which are characterised by non-ideal ordering of the molecules, in other words in areas with free space. For the molecules that are indicated by the coloured rings, the centre of mass position changes. Those molecules which undergo translational changes, simultaneously also undergo orientational changes of their legs.

\section{Dynamic Processes: Are they Random or Collective?}

In the previous paragraphs, we have described different dynamics (desorption-adsorption of legs), orientational/ conformational flexibility, and translation at the level of individual molecules. This description did not take into account the relation between the motion of individual molecules and the (lack of) dynamics of its surroundings. Desorption-readsorption phenomena of individual legs occur both in a non-correlated and correlated i.e. adjacent molecules show simultaneous dynamics) fashion. A reorientation of the legs (e.g. a five-star that 'rotates') always goes together with the desorption or reorientation of legs of adjacent molecules. Not surprisingly, quite some conformational dynamics occur in the area of the translationally mobile molecules. The conclusion to draw is that the conformational or translational motion of a given molecule will favour simultaneous dynamics of adjacent molecules.

\section{Inducing Dynamics at the Liquid/ Solid Interface}

It is well-known that dynamics can be induced at the liquid/solid interface. Lightresponsive molecules such as azobenzenes can undergo a trans-cis isomerisation at the liquid/solid interface upon irradia-

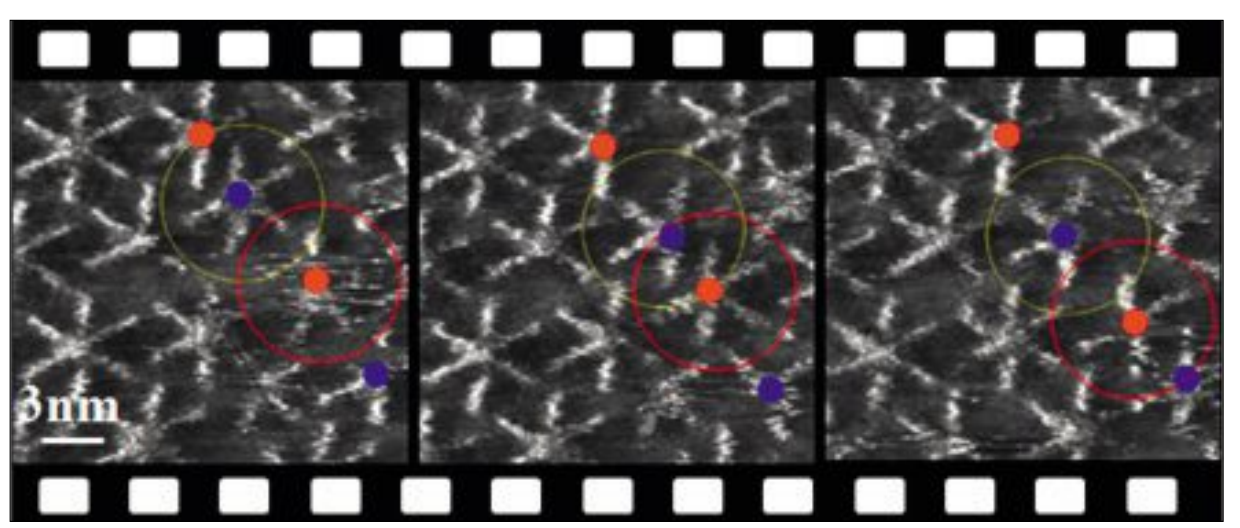

Fig. 6. Sequence of STM images zooming in on conformational and translational events in the monolayer of 1 at the liquid/solid interface. The time gap between the frames is 26 and 68 seconds, respectively. Coloured disks and circles indicate the centre and periphery of a number of selected molecules, respectively. 
tion, leading to a structural change in the monolayer or desorption. ${ }^{[48]}$ Alternatively, upon adding certain reagents to the liquid/ solid interface, reactions can be induced, which was elegantly demonstrated by Samorì et al. ${ }^{[55]}$ In all these cases, it is hard to establish unequivocally where the reaction really takes place: on the surface itself, or in the liquid phase. While this might be a matter of academic interest, such experiments show that it is possible to influence the surface composition at the liquid/solid interface. Recently, it was shown that using the concept of reactivity at the liquid/ solid interface, it is possible to form 2D porous polymers, also called covalent organic frameworks. ${ }^{[13,14]}$ The beauty of this approach is that the reactions involved are reversible, leading to highly regular networks. This might turn out to be an advantage compared to reactions that require a strict pre-organisation of the reagents at the liquid/solid interface, such as for the polymerisation of diacetylenes. ${ }^{[56]}$ On the other hand, some of these reactions can be initiated by the STM tip. Aono et al., and later our group, demonstrated that one can use the STM tip as a local electrode to induce the polymerisation of pre-organised diacetylenes. ${ }^{[57,58]}$

We recently investigated a system where applying momentary voltage pulses to the STM tip induced a major structural rearrangement in the monolayer. ${ }^{[59]}$ In this case, the STM tip does not induce a chemical reaction but can reversibly switch the monolayer into different phases. Indeed, experiments carried out at different tunneling conditions indicate that the supramolecular network formed by $\mathrm{PQPClO}_{4}$ molecules (Fig. 7A) at the 1-octanoic acid/ HOPG interface is extremely sensitive to electric parameters and could be manipulated by varying the substrate bias as well as by applying momentary voltage pulses to the STM tip. Thus, at a given set of tunneling parameters, either $\alpha$ or $\beta$ phase could be observed although there is more preference for the denser and disordered $\beta$ phase. In fact, the $\beta$ phase survives on the surface for longer times as long as no electric manipulation is carried out. Essentially, a significant change in the substrate bias $(\geq 300$ $\mathrm{mV}$ ) triggers the phase change. The same transformation can also be effected by applying $3.5 \mathrm{~V}(10 \mu \mathrm{s})$ pulses to the STM tip (Fig. 7B). The switching success rate per tip voltage pulse depends on the detailed configuration of the STM tip apex and it is rarely $100 \%$. Apart from the two phases mentioned above, a third phase ( $\gamma$ phase) also exists which dynamically transforms into the $\beta$ phase as a function of time and appears to be driven by an entropic gain (Fig. 7D). The course of $\gamma$ to $\beta$ phase transformation could be followed on the surface by recording time-dependent STM images.

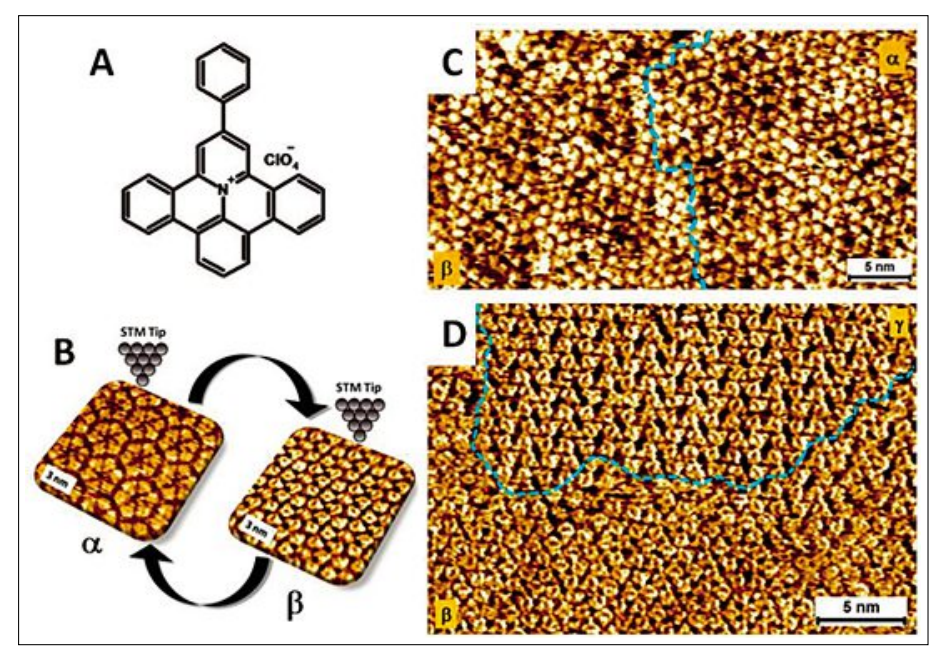

Fig. 7. A) Molecular structure of PQP$\mathrm{ClO}_{4}$. B) A schematic illustration of electric field induced reversible transition in the monolayer of PQP$\mathrm{ClO}_{4} . \mathrm{C}$ ) and D) STM images showing the coexistence of the $\alpha$ and $\beta(B)$ as well as $\beta$ and $\gamma(C)$ networks of $\mathrm{PQPClO}_{4}$. Dashed blue lines represent the domain boundaries.

Such time-dependent transformation of $\gamma$ phase to the $\beta$ phase is indicative of substantial surface dynamics in this charged monolayer.

In conclusion, by describing key examples from our research we have demonstrated that one can induce and visualise various kinds of dynamic processes at the liquid/solid interface namely conformational, adsorption-desorption, and translational dynamics with molecular precision. Furthermore, we also illustrated that it is possible to induce dynamics at the liquid/ solid interface by carrying out electric field manipulation. It would be interesting to see how some of these dynamic processes are influenced by changing the solvent or temperature. This will further develop our understanding of the dynamics of molecules in self-assembled monolayers, bringing insight into solvation at the liquid/solid interface.

\section{Acknowledgments}

This work is supported by KU Leuven through GOA 2006/2, the Institute of Promotion of Innovation by Science and Technology in Flanders (I.W.T.), the Fund of Scientific Research Flanders (FWO), and the Belgian Federal Science Policy Office through IAP6/27. In particular we thank the groups of Prof. E. W. Meijer and Prof. A. P. H. J. Schenning from TUEindhoven, and Prof. Müllen from MPI für Polymerforschung for very fruitful collaborations. In addition, also Prof. A. Gesquière and Prof. M. M. Abdel Mottaleb are acknowledged for their contributions.

Received: November 18, 2011

[1] G. C. McGonical, R. H. Bernhardt, D. J. Thomson, Appl. Phys. Lett. 1990, 57, 28.

[2] J. P. Rabe, S. Buchholz, Science 1991, 253, 424.

[3] T. Yokoyama, S. Yokoyama, T. Kamikado, Y. Okuno, S. Mashiko, Nature 2001, 413, 619.

[4] M. Böhringer, K. Morgenstern, W.-D. Schneider, R. Berndt, F. Mauri, A. De Vita, R. Car, Phys. Rev. Lett. 1999, 83, 324.

[5] A. Dmitriev, N. Lin, J. Weckesser, J. V. Barth, K. Kern, J. Phys. Chem. B 2002, 106, 6907.
[6] M. O. Lorenzo, C. Baddeley, C. J. Muryn, R. Raval, Nature 2000, 404, 376.

[7] J. Frommer, Angew. Chem., Int. Ed. 1992, 31, 1298.

[8] D. M. Cyr, B. Venkataraman, G. W. Flynn, Chem. Mater. 1996, 8, 1600.

[9] S. De Feyter, A. Gesquière, M. M. AbdelMottaleb, P. C. M. Grim, F. C. De Schryver, C. Meiners, M. Sieffert, S. Valiyaveettil, K. Müllen, Acc. Chem. Res. 2000, 33, 520.

[10] M. Kunitake, U. Akiba, N. Batina, K. Itaya, Langmuir 1997, 13, 1607.

[11] Y. He, T. Ye, E. Borguet, J. Am. Chem. Soc. 2002, 124, 11964.

[12] S. Yoshimoto, N. Higa, K. Itaya, J. Am. Chem. Soc. 2004, 126, 8540.

[13] R. Tanoue, R. Higuchi, N. Enoki, Y. Miyasato, S. Uemura, N. Kimizuka, A. Z. Stieg, J. K. Gimzewski, M. Kunitake, ACS Nano 2011, 5, 3923.

[14] J. F. Dienstmaier, A. M. Gigler, A. J. Goetz, P. Knochel, T. Bein, A. Lyapin, S. Reichlmaier, W. M. Heckl, M. Lackinger, ACS Nano 2011, 5, 9737.

[15] S. Berner, M. Brunner, L. Ramoino, H. Suzuki, H.-J. Guntherodt, T. A. Jung, T. A. Chem. Phys. Lett. 2001, 348, 175.

[16] M. Schunack, T. R. Linderoth, F. Rosei, E. Laegsgaard, I. Stensgaard, F. Besenbacher, Phys. Rev. Lett. 2002, 88, 156102.

[17] H. Yanagi, H. Mukai, K. Ikuta, T. Shibutani, T. Kamikado, S. Yokoyama, S. Mashiko, Nano Lett. 2002, 2, 601.

[18] J. Weckesser, J. V. Barth, K. Kern, J. Chem. Phys. 1999, 43, 5351

[19] R. Otero, F. Hummelink, F. Sato, S. B. Legoas, P. Thostrup, E. Laegsgaard, I. Stensgaard, D. D. Galvao, F. Besenbacher, Nature Mater. 2004, 3 , 779.

[20] J. A. Miwa, S. Weigels, H. Gersen, F. Besenbacher, F. Rosei, T. R. Linderoth, J. Am. Chem. Soc. 2006, 128, 3164.

[21] G. Schull, L. Douillard, C. Fiorini-Debuisschert, F. Charra, F. Mathevet, D. Kreher, A.-J. Attias, Adv. Mater. 2006, 18, 2954.

[22] G. Schull, L. Douillard, C. Fiorini-Debuisschert, F. Charra, F. Mathevet, D. Kreher, A.-J. Attias, Nano Lett. 2006, 6, 1360.

[23] S. M. Hou, T. Sagara, D. C. Xu, T. R. Kelly, E. Ganz, Nanotechnology 2003, 14, 566.

[24] N. Wintjes, D. Bonifazi, F. Y. Cheng, A. Kiebele, M. Stohr, T. A. Jung, Angew. Chem., Int. Ed. 2007, 46, 4089.

[25] M. Wahl, M. Stohr, H. Spillmann, T. A. Jung, L. Gade, Chem. Commun. 2007, 13, 1349.

[26] T. A. Jung, R. R. Schlittler, J. K. Gimzewski, Nature 1997, 386, 696.

[27] L. J. Scherer, L. Merz, E. C. Constable, C. E. Housecroft, M. Neuburger, B. A. Hermann, $J$. Am. Chem. Soc. 2005, 127, 4033. 
[28] K. B. Kim, K. E. Plass, A. J. Matzger, Langmuir 2005, 21, 647.

[29] S. Weigelt, C. Busse, L. Petersen, E. Rauls, B. Hammer, K. V. Gothelf, F. Besenbacher, T. R. Linderoth, Nature Mater. 2006, 5, 112.

[30] C. Busse, S. Weigelt, L. Petersen, E. Laegsgaard, F. Besenbacher, T. R. Linderoth, A. H. Thomsen, M. Nielsen, K. V. Gothelf, $J$ Phys. Chem. B 2007, 111, 5850.

[31] M. Lingenfelder, G. Tomba, G. Costantini, L. C. Ciacchi, A. De Vita, K. Kern, Angew. Chem. Int. Ed. 2007, 46, 4492.

[32] T. A. Jung, R. R. Schlittler, J. K. Gimzewski, H. Tang, C. Joachim, Science 1996, 271, 181.

[33] M. Bohringer, K. Morgenstern, W. D. Schneider, R. Berndt, Angew. Chem. Int. Ed. 1999, 38, 821 .

[34] H. Yanagi, K. Ikuta, H. Mukai, T. Shibutani, Nano Lett. $2002,2,951$.

[35] J. A. A. W. Elemans, M. C. Lensen, J. W Gerritsen, H. van Kempen, S. Speller, R. J. M Nolte, A. E. Rowan, Adv. Mater. 2003, 15, 2070.

[36] R. Moresco, Phys. Rep. 2004, 399, 175.

[37] S. J. H. Griessl, M. Lackinger, F. Jamitzky, T. Markert, M. Hietschold, W. A. Heckl, Langmuir 2004, 20, 9403.

[38] N. Lorente, R. Rurali, H. Tang, J. Phys.: Condens. Matter 2005, 17, S1049.

[39] M. Stohr, M. Wahl, H. Spillmann, L. H. Gade, T. A. Jung, Small 2007, 3, 1336.
[40] J. J. de Jonge, M. A. Ratner, S. W. de Leeuw, J. Phys. Chem. C 2007, 111, 3770.

[41] C. Meier, K. Landfester, D. Kunzel, T. Markert, A. Gross, U. Ziener, Angew. Chem. Int. Ed. 2008, 47, 3821

[42] Y. L. Yang, Q. L. Chan, X. J. Ma, K. Deng, Y. T. Shen, X. Z. Feng, C. Wang, Angew. Chem. Int. Ed. 2006, 45, 6889.

[43] A. Stabel, R. Heinz, F. C. De Schryver, J. P. Rabe, J. Phys. Chem. 1995, 99, 505.

[44] P. Samorì, K. Müllen, J. P. Rabe, Adv. Mater. 2004, 16, 1761 .

[45] G. M. Florio, J. E. Klare, M. O. Pasamba, T. L. Werblowsky, M. Hyers, B. J. Berne, M. S. Hybertsen, C. Nuckolls, G. W. Flynn, Langmuir 2006, 22, 10003.

[46] B. A. Hermann, L. J. Scherer, C. E. Housecroft, E. C. Constable, Adv. Funct. Mater. 2006, 16, 221.

[47] D. Rohde, C. Y. Yan, H. J. Yan, L. J. Wan Angew. Chem. Int. Ed. 2006, 45, 3996.

[48] P. Vanoppen, P. C. M. Grim, M. Rücker, S. De Feyter, G. Moessner, S. Valiyaveetil, K. Müllen, F. De Schryver, J. Phys. Chem. 1996, 100, 19636.

[49] A. Gesquière, M. M. Abdel-Mottaleb, S. De Feyter, F. C. De Schryver, M. Sieffert, K. Müllen, A. Calderone, R. Lazzaroni, J.-L. Brédas, Chem. Eur. J. 2000, 6, 3739.
[50] A. Gesquière, M. Abdel-Mottaleb, F. C. De Schryver, Langmuir 1999, 15, 6821.

[51] F. Stevens, T. P. Beebe, Langmuir 1999, 15, 6884.

[52] Ž. Tomović, J. van Dongen, S. J. George, H. $\mathrm{Xu}, \mathrm{W}$. Pisula, P. Leclère, S. De Feyter, E. W. Meijer, A. P. H. J. Schenning, J. Am. Chem. Soc. 2007, 129, 16190.

[53] H. Xu, A. Minoia, Ž. Tomović, R. Lazzaroni, E. W. Meijer, A. P. H. J. Schenning, S. De Feyter, ACS Nano 2009, 3, 1016.

[54] H. Xu, M. Wolffs, Ž. Tomović, E. W. Meijer, A. P. H. J. Schenning, S. De Feyter, CrystEngComm 2011, 13, 5584.

[55] A. Ciesielski, S. Lena, S. Masiero, G. P. Spada, P. Samorì, Angew. Chem. Int. Ed. 2010, 49, 1.

[56] P. C. M. Grim, S. De Feyter, A. Gesquière, P. Vanoppen, M. Rücker, S. Valiyaveettil, G. Moessner, K. Müllen, F. C. De Schryver, Angew. Chem. Int. Ed. 1997, 36, 2601.

[57] Y. Okawa, M. Aono, Nature 2001, 409, 683.

[58] A. Miura, S. De Feyter, M. M. S. AbdelMottaleb, A. Gesquière, P. C. M. Grim, G. Moessner, M. Sieffert, M. Klapper, K. Müllen, F. C. De Schryver, Langmuir 2003, 19, 6474.

[59] K. S. Mali, D. Wu, X. Feng, K. Müllen, M. Van der Auweraer, S. De Feyter, J. Am. Chem. Soc. 2011, 133, 5686 . 